\title{
Approaching Free Fall on Two Degrees of Freedom: Simultaneous Measurement of Residual Force and Torque on a Double Torsion Pendulum
}

\author{
M. Bassan, ${ }^{1,2,{ }^{*}}$ A. Cavalleri, ${ }^{3}$ M. De Laurentis,${ }^{6,7}$ F. De Marchi,${ }^{1,2, \dagger}$ R. De Rosa,${ }^{6,7}$ L. Di Fiore, ${ }^{7, \ddagger}$ R. Dolesi,${ }^{4,5}$ N. Finetti,${ }^{8,9}$ \\ F. Garufi, ${ }^{6,7}$ A. Grado, ${ }^{10,7}$ M. Hueller, ${ }^{4,5}$ L. Marconi ${ }^{11,9,8}$ L. Milano, ${ }^{6,7}$ G. Pucacco, ${ }^{1,2}$ R. Stanga, ${ }^{11,9}$ M. Visco, ${ }^{12,2}$ \\ S. Vitale, ${ }^{4,5}$ and W. J. Weber, \\ ${ }^{1}$ Dipartimento di Fisica, Università di Roma "Tor Vergata," I-00133 Roma, Italy \\ ${ }^{2}$ INFN_Sezione Roma2, I-00133 Roma, Italy \\ ${ }^{3}$ CNR-Istituto di Fotonica e Nanotecnologie, - Fondazione Bruno Kessler, 38123 Povo (TN), Italy \\ ${ }^{4}$ Dipartimento di Fisica, Università di Trento, I-38050 Povo (TN), Italy \\ ${ }^{5}$ INFN-TIFPA, 38050 Povo (TN), Italy \\ ${ }^{6}$ Dipartimento di Fisica, Università di Napoli "Federico II," I-80126 Napoli, Italy \\ ${ }^{7}$ INFN-Sezione di Napoli, I-80126 Napoli, Italy \\ ${ }^{8}$ Dipartimento di Scienze Fisiche e Chimiche, Università degli Studi dell'Aquila, I-67100 l'Aquila, Italy \\ ${ }^{9}$ INFN-Sezione di Firenze, I-50019 Firenze, Italy

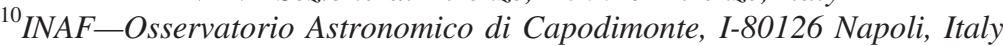 \\ ${ }^{11}$ Dipartimento di Fisica ed Astronomia, Università degli Studi di Firenze, I-50019 Firenze, Italy \\ ${ }^{12}$ INAF-Istituto di Astrofisica e Planetologia Spaziali, I-00133 Roma, Italy
}

(Received 28 November 2015; published 5 February 2016)

\begin{abstract}
A torsion pendulum with 2 soft degrees of freedom (DOFs), realized by off-axis cascading two torsion fibers, has been built and operated. This instrument helps characterize the geodesic motion of a test mass for LISA Pathfinder or any other free-fall space mission, providing information on cross talk and other effects that cannot be detected when monitoring a single DOF. We show that it is possible to simultaneously measure both the residual force and the residual torque acting on a quasifree test mass. As an example of the investigations that a double pendulum allows, we report the measurement of the force-to-torque cross talk, i.e., the amount of actuation signal, produced by applying a force on the suspended test mass, that leaks into the rotational DOF, detected by measuring the corresponding (unwanted) torque.
\end{abstract}

DOI: 10.1103/PhysRevLett.116.051104

Introduction.-Achieving geodesic motion is a key benchmark for the LISA-class [1], space based gravitational wave $(\mathrm{GW})$ experiments. The possibility of measuring small, GW induced changes in the distance between the test masses (TMs) crucially depends on the quality of free fall of these masses, i.e., on the experimenter's ability to reduce all external sources of noise forces that might alter the free motion along geodesic lines. The space mission LISA Pathfinder (LPF) [2], launched in 2015 and operational in early 2016, has as its top scientific goal the proof of a differential acceleration measurement between freefalling test masses, inside a single spacecraft, at the level of $30 \mathrm{fm} \mathrm{s}^{-2} \mathrm{~Hz}^{-1 / 2}$ at $1 \mathrm{mHz}$, a thousand times lower than previously achieved [3,4]. Extensive ground-based testing was required on the proof mass and its read-out prior to launch, to investigate, mitigate, and fight all possible sources of noise (thermal, electric, magnetic, etc.) and coupling to the environment that might affect the apparatus. For this task, the torsion pendulum has been the instrument of choice for measuring small forces and torques: the suspension fiber, while balancing the vertical pull of gravity, exerts a very weak restoring torque. This is, still today, the best approximation to a free motion along one degree of freedom (DOF): the fiber rotation around its axis.
Different kinds of pendulums have been devised and operated, where the quasifree mode is either the rotation along the TM axis $[5,6]$ or the motion along a large arc that approximates, for small angles, a linear translation [7]. A pendulum of the first kind is suitable for investigating residual torque, while the second is sensitive to force. These instruments have set important upper limits on surface forces and torques that could affect the geodesic motion of LISA TMs $[8,9]$. The obvious limitation of these experiments lies in the single "soft" DOF available for testing, while the test mass in LPF will be free (or under feedback control) along all 6 DOFs. It is naturally desirable, for the purpose of better characterizing the behavior of a test mass in geodesic motion, to have an instrument that behaves as almost free along more than one degree of freedom. This would allow for the investigation of possible spurious couplings between motion on different DOFs, nondiagonal residual couplings between TM and read-out (the so called stiffnesses), read-out and actuation cross talk from one DOF to the other, and so on. Such tests are crucial for the drag-free operation [10] on LPF, where the TMs will move freely in the sensitive direction but will be under feedback control in the remaining five directions. In this Letter we report on the operation of a twofold torsion pendulum, 
named PETER [11], designed to simulate on ground (down to a few millihertz) the free-fall condition on 2 DOFs in the very low frequency band of interest for space-born gravitational wave detectors. The apparatus was developed as a ground-testing facility for the characterization of the readout-actuation system of the LPF mission. Various in-flight activities for LPF and eLISA, like measurements of charge, dc electrostatic biases, and magnetic effects, will simultaneously introduce forces and torques on different axes that require simultaneous detection and calibration. Finally, the simultaneous measurement of forces and torques on a TM allows us to probe different length scales for the spatial distribution of effects like, for instance, stray electrostatic and thermal fluctuations, with torques arising in variations across a single TM face, while net forces will arise in variations between opposing TM faces. Other twofold pendulums have been developed $[12,13]$, but with a different goal, mode, and frequency of operation; PETER is, to our knowledge, the first apparatus where quasifree-fall and residual forces on one TM can be measured for 2 different DOFs.

Instrument description. - We have developed a two-stage torsion pendulum where, by cascading two torsion fibers, we achieve soft motion along 2 DOFs: rotation along a vertical axis passing through the center of mass and translation along a horizontal direction. The apparatus was first developed and operated at the INFN laboratory in Florence and then transferred to the Gravitational Physics Laboratory in Naples, where we can exploit better environmental conditions and where the facility is now fully operative. We report here on the present results obtained with this apparatus, and on the first simultaneous measurements of residual force and torque on the test mass and of force-totorque cross-couplings. In this instrument, a cubic TM is suspended through a double stage system with two torsion fibers in cascade (see Fig. 1): the lower fiber allows this mass

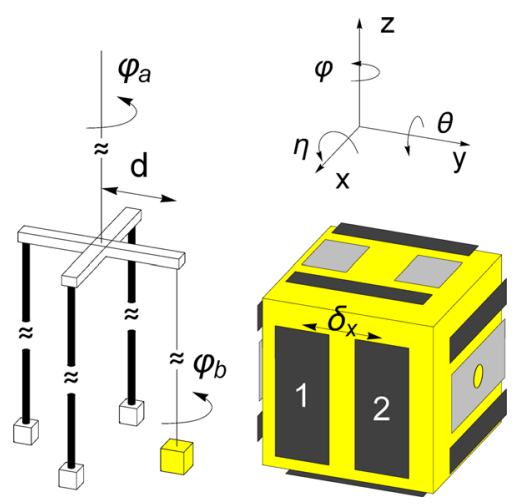

FIG. 1. A sketch of the PETER pendulum. A diagram of the GRS electrodes, surrounding the TM, is also shown: the gray electrodes feed ac bias while the black ones are for sensing and actuation. Labels 1 and 2 indicate two of the four electrodes sensing $x$ and $\varphi$ DOFs: each one is paired in a bridge with an identical electrode on the opposite side. to be almost free in rotation around its symmetry vertical axis. The fiber hangs from the tip of one arm (of length $d=15 \mathrm{~cm}$ ) of a crossbar that is, in turn, suspended by an upper fiber that is secured to the vacuum enclosure, i.e., to the lab reference frame. Torsion of this upper fiber allows almost free motion of the suspended system, including the test mass, along an arc of a $30 \mathrm{~cm}$ diameter circumference: for small torsion angles and for all practical purposes, this can be considered a translational motion. Dummy loads hang, through rigid rods, from the three other arms of the crossbar, with a similar mass distribution, in order to minimize undesired quadrupole couplings. The complete dynamics of a double pendulum is complex and was detailed in a dedicated paper [14]. However, when only the $2 \mathrm{soft}$ DOFs are considered, this dynamics can be reduced to a much simpler form, described by two coupled equations in the variables $\varphi_{a}, \varphi_{b}$ describing the torsion angles of the upper ( $a=$ above) and lower ( $b=$ below) fibers:

$$
\begin{aligned}
\left(I_{a}^{\prime}+I_{b}\right) \ddot{\varphi_{a}}+I_{b} \ddot{\varphi_{b}}+\gamma_{a} \dot{\varphi}_{a}+k_{a} \varphi_{a} & =F_{b} d+\tau_{a}+\tau_{b}, \\
I_{b} \ddot{\varphi}_{a}+I_{b} \ddot{\varphi_{b}}+\gamma_{b} \dot{\varphi}_{b}+k_{b} \varphi_{b} & =\tau_{b},
\end{aligned}
$$

where $I_{j}, k_{j}$, and $\gamma_{j}$ represent the moment of inertia, the elastic constant, and the dissipation factor of the $j$ th pendulum $(j=a, b)$, and $\tau_{j}, F_{j}$ the external torque and force acting on each load mass. The crossbar inertia is actually modified: $I_{a}^{\prime}=I_{a}+d^{2} m_{b}\left(1+m_{b} / m_{a}\right)$, by the presence of the test mass (see Ref. [14] for details). Equivalently, the dynamics can be described by two coordinates of the TM, directly measured by the read-out, i.e., its rotation angle $\varphi$ around the $z$ axis and the displacement along the $x$ axis. These alternate variables are related to the two torsion angles by

$$
x=\varphi_{a} d \quad \varphi=\varphi_{a}+\varphi_{b} .
$$

The TM is a hollow $\mathrm{Al}$ cube with a $46 \mathrm{~mm}$ edge plus a shaft $(81.5 \mathrm{~mm}$ long) that connects to the fiber and provides electrical insulation. The TM is enclosed in the gravitational reference sensor (GRS) [15], a hollow metal box padded with 16 electrodes: 12 of these are arranged in six capacitive bridges, and permit us to monitor the motion of the TM along all of its translational and rotational DOFs. The same electrodes can be individually biased with control voltages in order to apply forces or torques. The GRS (we use the LPF engineering model) can be micropositioned in 5 degrees of freedom; additionally, the suspension point of the $a$ fiber can be raised and rotated: this allows us to align the TM at the center of the GRS, and parallel to its walls. Transduction and actuation is operated by a read-out and control electronics unit, specially developed at ETH [16]. The apparatus is also equipped with two additional readouts-an autocollimator and an optical read-out system $[17,18]$ — to provide independent measurements of the test mass position along the 2 soft DOFs. Further details on the apparatus can be found in Refs. [19-21]. 


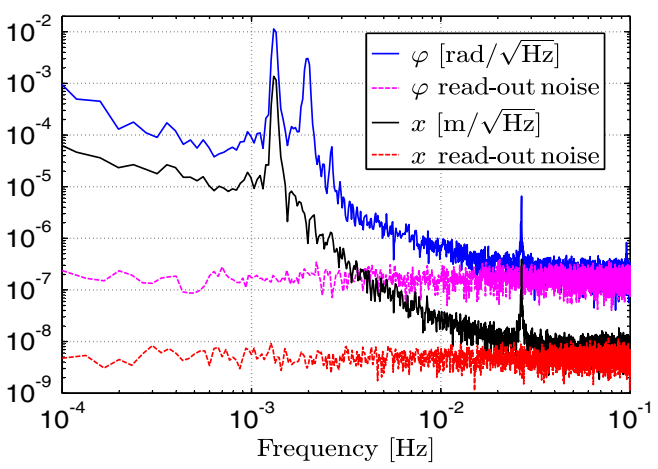

FIG. 2. Amplitude spectral density of the translational ( $x$, black) and rotational $(\varphi$, blue) DOFs of the double pendulum. The useful "free-fall" band extends above the resonances (1.3 and $2.0 \mathrm{mHz}$ ) till $30 \mathrm{mHz}$, where read-out noise becomes dominant in both spectra. The peak near $25 \mathrm{mHz}$ results from the beats of a swinging doublet mode (see Ref. [14]).

Experimental results. - In Fig. 2 we report the measured spectrum of the 2 soft DOFs as measured by the GRS. According to Eq. (1) the $\varphi$ spectrum shows two resonances associated with the two fibers, while $x$ only shows the resonance peak associated with the upper fiber. We use the time series $x(t)$ and $\varphi(t)$ and their derivatives to solve the equations of motion (1) and compute force and torque acting on the TM, as shown in Fig. 3. Considering that a torque acting on the crossbar $\left(\tau_{a}\right)$ cannot be distinguished, in our assumption, from a force acting on the TM center of mass $\left(F_{b} d\right)$, we are actually assessing an upper limit to $F_{b}$. In this way, we can assess upper limits on both force and torque simultaneously acting on our TM, down to a few millihertz. A few preliminary measurements were performed to calibrate the apparatus and to improve estimates of the parameters (like the moments of inertia) that are relevant for the characterization of the apparatus [e.g., for the correct integration of Eq. (1)]. This allowed us to obtain an absolute calibration of the applied force and torque that agrees, within $10 \%$, with a simplified, infinite plate model of the GRS electrostatics. The model, taking into account the electrode geometry and the TM-GRS relative position, is derived from Ref. [22], simplified with a series expansion

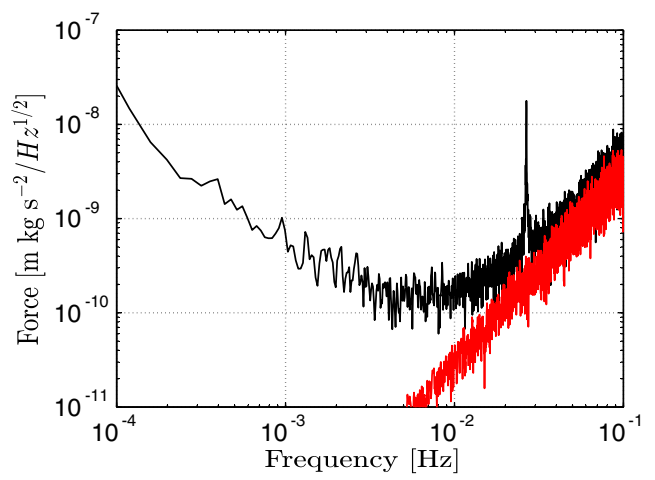

to second order in the electrode capacitance derivatives. The agreement is within the uncertitude of the model, which deviates up to $20 \%$ from a finite element analysis of the GRS [23].

As an example of how PETER can provide information relevant for the physics investigated by LPF, we describe here the measurement of cross talk (CT). We recall that the test mass will be in free fall along the $x$ axis, while under feedback control on the remaining DOFs: an undue cross talk of these feedback signals into the $x$ channel would spoil the quality of geodesic motion. We have run several investigations of the force to torque $\mathrm{CT}$, with the TM in various off-center positions inside the GRS, by actuating with a sinusoidal force on the TM: we generate an ac bias with an amplitude that varies as $V_{\text {act }}=V_{0} \sqrt{\sin \left(\omega_{\text {act }} t\right)}$, applying it alternatively on each pair of electrodes. This produces a force (or torque, depending on the electrodes used)

$$
F(t)=\frac{1}{2} \sum_{i=1}^{4} \frac{\partial C_{i}}{\partial x} V_{0}^{2} \sin \left(\omega_{\mathrm{act}} t\right)
$$

where the $C_{i}$ represent the capacitances of the four $x$ electrodes to the TM (see Fig. 1). The GRS is moved around the TM with motorized translation stages along the horizontal plane according to a regular $(x, y)$ pattern. For each grid point we collected data in stretches a few hours long and extracted, by coherent detection, the force $(F)$ and the torque $(\tau)$ acting on the TM at the actuation frequency. The cross talk is defined as the ratio of applied force to the detected torque: i.e., $C T_{F \rightarrow \tau}=\tau /\left(F \delta_{x}\right)$, where the distance between the center of the $x$ electrodes $\delta_{x}=2.15 \mathrm{~cm}$ is the scale factor that converts the force into a torque. Using the model, this cross talk is given by

$$
C T_{F \rightarrow \tau}=\frac{y}{\delta_{x}}+\frac{h_{x}}{C_{0} \delta_{x}}\left(\frac{\partial^{3} C}{\partial x \partial \varphi^{2}}\right)_{x=\varphi=0} x \varphi,
$$

where $h_{x}=4 \mathrm{~mm}$ is the gap between the TM and the GRS along the $x$ axis and $C_{0}$ is the value of the $x$ electrode capacitance when the TM is centered in the GRS.

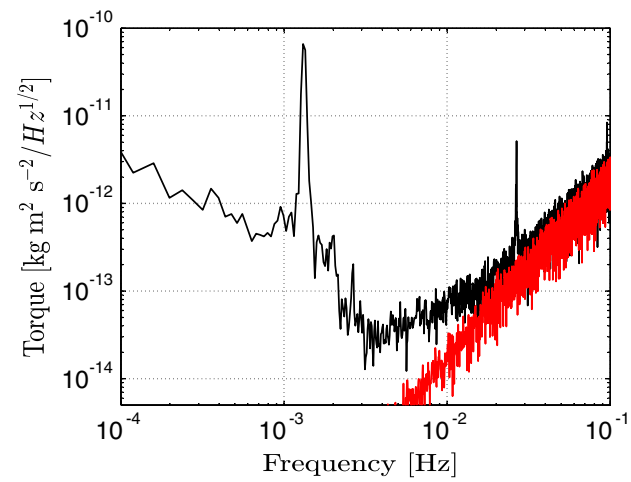

FIG. 3. Spectra of residual force (left panel) and torque (right panel) acting on the test mass. Read-out noise is shown in red. 


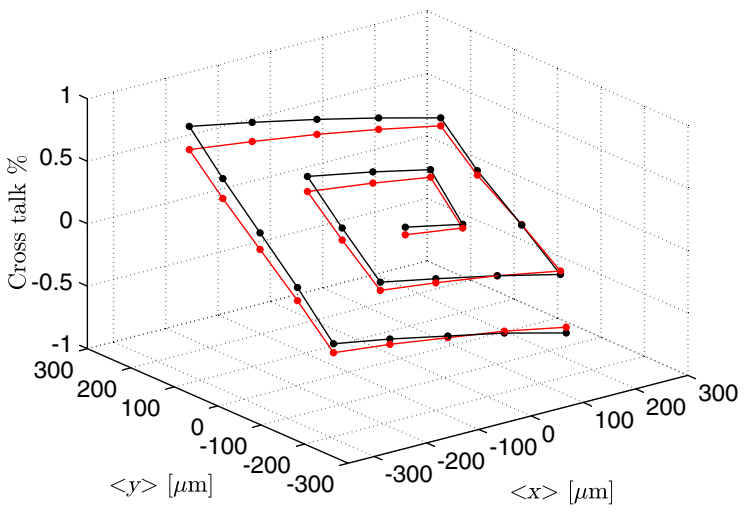

FIG. 4. Cross talk measurement (the black line) vs offset mean position (center of oscillation) inside the GRS. Data of a typical run are compared with the model predictions (the red line).

In Fig. 4, we report, as an example, the results from one of the $C T_{F \rightarrow \tau}$ measurement runs and performed by moving the GRS along a spiral pattern in $x$ and $y$, starting with the TM centered inside the GRS, for a total of 25 positions. The total measurement time is, in the case shown, $50 \mathrm{~h}$. The experimental data are compared to the analytical model. These measured values proved to be quite repeatable from run to run; they lie on a regular surface that is well described by $2 \mathrm{D}$ second order equations that well match the model. The agreement between measured and expected values is quite good: the maximum observed disagreement between data and model predictions is $0.2 \%$ in CT on the entire science mode range of the GRS $( \pm 200 \mu \mathrm{m})$. This agreement is unaffected by the previously mentioned systematics, which cancels out when the CT is computed as a ratio of the measured quantities. These tests thus confirm the model of the expected cross talk term, with an applied $x$ force also producing a net torque when the TM is off center in either $x$ or $y$ with respect to the polarized electrodes.

Conclusions.-We reported on the first measurements from a twofold torsion pendulum designed to simulate on ground the free-fall condition on 2 DOFs in the very low frequency band (down to $10^{-4} \mathrm{~Hz}$ ) of interest for LPF and space-born GW detectors. Extension of this instrument concept to 3 soft DOFs (cascading two crossbars) is, in principle, straightforward [19]. We produced the first simultaneous measurement of both force and torque acting on the TM. This allows us to perform two-dimensional tests that cannot be achieved with a single stage torsion pendulum. As an example, we reported on measurements of force-to-torque actuation cross talk for the GRS. These data show that the measured CT is very close to expectations. In particular, the measured CT is below $0.1 \%$ when the TM is centered (in 6 DOFs) in the GRS. It is below $0.3 \%$ in a $\pm 100 \mu \mathrm{m}$ interval around the center, and below $0.8 \%$ in the whole high sensitivity range $( \pm 200 \mu \mathrm{m})$. These measured CT values agree with the model predictions within $0.2 \%$. These values are within the specifications for LPF. The apparatus is now in operation, completing the experimental characterization in 2 DOFs of the LISAPathfinder GRS. It will also serve as a test bench for verifying procedures during the mission operations. This new measurement technique will be useful for the ground testing of the future space gravitational wave detectors like eLISA and for other precision space and ground-based experiments.

*Corresponding author.

bassan@roma2.infn.it

'Present address: DIMA, Sapienza, Università di Roma, 00184 Roma, Italy.

Corresponding author. difiore@na.infn.it

${ }^{\S}$ Present address: INO_CNR, 50125 Firenze, Italy.

[1] eLISA Consortium, arXiv:1305.5720.

[2] F. Antonucci et al., Classical Quantum Gravity 29, 124014 (2012).

[3] R. Floberghagen, M. Fehringer, D. Lamarre, D. Muzi, B. Frommknecht, C. Steiger, J. Piñeiro, and A. da Costa, J. Geodes. 85, 749 (2011).

[4] B. Sheard, G. Heinzel, K. Danzmann, D. A. Shaddock, W. M. Klipstein, and W. M. Folkner, J. Geodes. 86, 1083 (2012).

[5] L. Carbone, A. Cavalleri, R. Dolesi, C. D. Hoyle, M. Hueller, S. Vitale, and W. J. Weber, Phys. Rev. Lett. 91, 151101 (2003).

[6] M. Bassan, F. De Marchi, L. Marconi, G. Pucacco, R. Stanga, and M. Visco, Phys. Lett. A 377, 1555 (2013).

[7] A. Cavalleri et al., Classical Quantum Gravity 26, 094017 (2009).

[8] L. Carbone, G. Ciani, R. Dolesi, M. Hueller, D. Tombolato, S. Vitale, W. J. Weber, and A. Cavalleri, Phys. Rev. D 75, 042001 (2007).

[9] L. Carbone, A. Cavalleri, G. Ciani, R. Dolesi, M. Hueller, D. Tombolato, S. Vitale, and W. J. Weber, Phys. Rev. D 76, 102003 (2007).

[10] B. Lange, AIAA J. 2, 1590 (1964).

[11] Italian acronym for "PEndulum Translational and Rotational".

[12] Z. B. Zhou, L. Liu, H. B. Tu, Y. Z. Bai, and J. Luo, Classical Quantum Gravity 27, 175012 (2010).

[13] H. B. Tu, Y. Z. Bai, Z. B. Zhou, L. Liu, L. Cai, and J. Luo, Classical Quantum Gravity 27, 205016 (2010).

[14] F. De Marchi, G. Pucacco, M. Bassan, R. De Rosa, L. Di Fiore, F. Garufi, A. Grado, L. Marconi, R. Stanga, F. Stolzi, and M. Visco, Phys. Rev. D 87, 122006 (2013).

[15] R Dolesi et al., Classical Quantum Gravity 20, S99 (2003).

[16] L. Gan, D. Mance, and P. Zweifel, Sens. Actuators, A 167, 574 (2011).

[17] F. Acernese, E. Calloni, R. De Rosa, L. Di Fiore, and L. Milano, Classical Quantum Gravity 22, S279 (2005).

[18] R. De Rosa, L. Di Fiore, F. Garufi, A. Grado, A. La Rana, and L. Milano, Astropart. Phys. 34, 394 (2011). 
[19] L. Marconi, Ph.D. thesis, Università di Firenze, 2006.

[20] R. Stanga, L. Marconi, C. Grimani, M. Bassan, G. Pucacco, E. Reali, R. Simonetti, and N. Finetti, J. Phys. Conf. Ser. 154, 012032 (2009).

[21] L. Marconi, R. Stanga, and M. Bassan, J. Phys. Conf. Ser. 363, 012049 (2012).
[22] J. Martijn Smit, LISA Pathfinder Project Documentation No. S2-SRO-TN-2001 2.1, 2006.

[23] N. Brandt and W. Fichter, J. Phys. Conf. Ser. 154, 012008 (2009). 